\title{
An adaptive disturbance rejection control scheme for voltage regulation in DC micro-grids
}

\author{
Unnikrishnan Raveendran Nair, Ramon Costa-Castelló \\ Institut de Robòtica i Informòtica industrial \\ Industrial, CSIC-UPC Llorens i Artigas 4-6, 08028 \\ Barcelona, Spain \\ uraveendran@iri.upc.edu,ramon.costa@upc.edu
}

\author{
Germán Andres Ramos \\ Departamento Ingeniera Eléctrica y Electrónica \\ Universidad Nacional de Colombia \\ Bogota, Columbia \\ garamosf@unal.edu.co
}

\begin{abstract}
Uncertain generation by renewable sources and load variations have resulted in adding energy storage systems in the grid to maintain grid parameters (voltage, frequency) within prescribed limits. The disturbances being non-deterministic in nature, the voltage regulation control by the storage systems relies mostly on dual loop architecture with an outer voltage and inner current loop. Improvement in controller dynamics can be achieved through feed forward of disturbance profile but at expense of additional sensors and communication in the grid. This work explores the application of an adaptive disturbance rejection control scheme for disturbance estimation (without using additional sensors) employing an extended state and proportional integral observer (PI+ESO). The proposed observer aim to achieve robust disturbance estimation under grid parameter uncertainty. The effectiveness of the proposed scheme over the conventional one will be put forward through $H_{\infty}$ and $\mathrm{H}_{2}$ norm analysis of the system. The design and simulation results of the proposed scheme will be presented in this work.

Index Terms-state estimation, grid compensation and control, storage systems control.
\end{abstract}

\section{INTRODUCTION}

The ever growing addition of renewable sources which are non dispatchable can cause variations in voltage and frequency profile of grid beyond permissible limits during supply-demand imbalance, as the generation is decoupled with the load demand [1]. These issues are overcome through the addition of energy storage systems (ESS) in the grid which cater to short and long term imbalances ensuring the power quality in the grid. These ESS smooths out the power profiles from the renewable sources, ensures energy balance and provide a degree of inertia in the grid having high penetration of renewable sources [2]-[4].

Power converters interfacing the ESS to grid allow control of power delivered or absorbed by ESS. An effective control of power converter is essential in ensuring power quality in the grid. The major issue in grids with high renewable penetration is the non-deterministic nature of its generation and load demand. As such defining a control strategy that is based on complete knowledge of the load and generation profile, to ensure power balance, requires extensive addition of sensors and high bandwidth communication. Such a control can be impractical [5]. Therefore the emphasis has to be on defining control strategies that rely on low bandwidth communication.

One of the widely used control architecture in voltage regulation of grids with sources connected through power converters is the dual loop architecture which employs an outer loop for voltage control and inner loop for current control [6]-[8]. In networks with multiple sources connected, the dual loop architecture is augmented with the droop control to ensure power distribution among the different sources based on their capacities [9]. Improving the dynamics of dual loop control can be achieved by feed forwarding the disturbance which can be variations in renewable generation or load profiles [10], [11].This again requires a complete knowledge of the disturbance through measurements which is impractical. As such another method that can be employed to improve controller dynamics is use of observers to estimate disturbance.

Providing an estimate of the disturbance generated by the loads, sources or modelling uncertainties improve the dynamics of control without relying on high bandwidth communication and extensive metering in the system. This can be achieved using a class of observers derived from the Luenberger observer like the Disturbance observer and Proportional integral observer [12]. Disturbance observers (DO) provide an estimation of disturbances using an approximate model of the disturbance acting on the system based on some prior knowledge of the system [13] [14]. These observers are also referred to as an extended state observer(ESO) as it considers disturbance as an additional state of the system. The proportional integral (PI) observer is a modification of the Luenberger observer which augments an integral part to the Luenberger structure. This observer provides a robust estimation of step disturbances under system uncertainties [12] [15]. In [16] an application of ESO is shown for input DC link voltage regulation of two level three phase converter. In the above work disturbance in the form of a load connected at the DC link of the converter is estimated using the observer to improve the voltage regulation. Another work which considers the use of observer in the voltage regulation problem for power converter connected system is [5]. Here a non linear observer 
system is employed in the voltage regulation of the DC bus voltage in a hybrid AC/DC system. The observer estimates the disturbance acting on the DC bus of the hybrid system and uses it as a feed forward in the control loop of the AC/DC power converter interfacing the $\mathrm{AC}$ side to $\mathrm{DC}$ side thereby achieving disturbance rejection.

In this work the authors consider the application of observer estimation in the voltage regulation of a local DC microgrid having renewable generation and storage capability in supercapacitors. The proposed control method will use an ESO along with a PI observer (PI+ESO) with the objective of providing disturbance estimation and robustness towards parameter uncertainty. The conventional dual loop architecture will be employed and aided by the disturbance estimation from $\mathrm{PI}+\mathrm{ESO}$ to achieve improved dynamic performance. The control architecture will henceforth be referred to as an adaptive disturbance rejection controller (ADRC). The proposed ADRC will be employed in the control loop of the DC-DC converter for supercapcitor (SC) as the SC ensures smooth voltage profiles under short term energy imbalances. The profiles of both sources and loads considered here are non deterministic in nature. In this work the design criteria of the ADRC scheme and the simulation will be presented. The improvement in performance of conventional dual loop architecture with the addition of PI+ESO will be assessed through system norms like $H_{\infty}$ and $H_{2}$. Results in robustness analysis to parameter uncertainty will also be provided.

The rest of the paper is outlined as follows. Section II presents the DC-DC converter used in the application and modelling of the same. In section III the ADRC scheme is presented along with the PI+ESO. The system equations and control law will be discussed in this section. In section IV the $H_{\infty}$ and $H_{2}$ norm based analysis of the proposed scheme is done. The design of ADRC for the specific case considered in this work and the simulation results will be outlined in section V. Finally the work will concluded in Section VI.

\section{DC-DC CONVERTER MODEL}

The DC-DC converter used in this work is shown in Fig.1 and is capable of two quadrant operation. This enables the converter to step up or down the voltage depending on the direction of power flow. The power electronic switches $S_{1}$ and $S_{2}$ are realised using IGBTs controlled by complementary gating signals with appropriate dead time between them. The inductance $l_{s c}$ facilitates the stepping up and stepping down of input-output voltage of the converter. The resistance $r_{s c}$ is the effective series resistance (ESR) of the inductor. The voltage source $v_{s c}$ represents the SC. In the proposed application the converter will function to ensure that $v_{b u s}$, the DC-grid voltage, remains constant under disturbances which will be defined later. The converter will be modelled considering the average waveforms of the systems devoid of higher order switching ripple. This is done using averaged voltage across IGBT $S_{2}$ given by $v_{c s c}=d^{\prime} v_{b u s}$ where $d^{\prime}=1-d$ with $d$ the duty ratio of the gate signals to $S_{2}$. The averaged model for the system is developed using the methodology proposed in

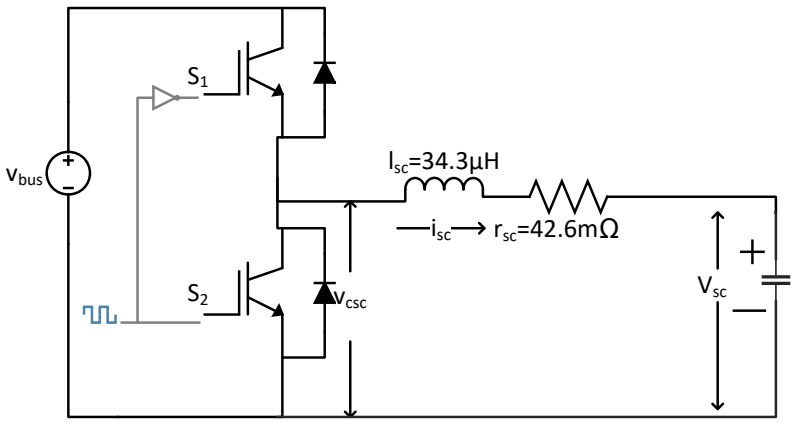

Fig. 1. DC-DC bidirectional power converter schematic.

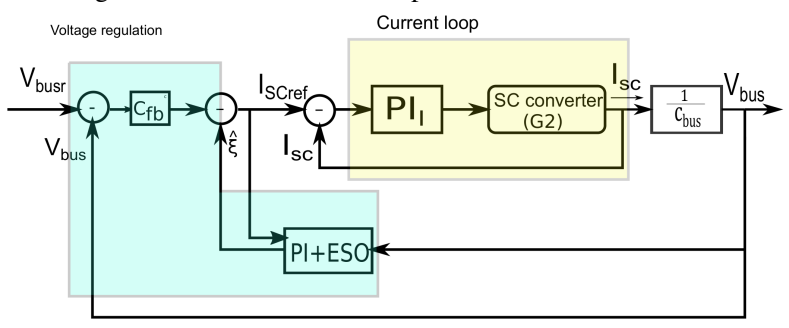

Fig. 2. ADRC scheme for voltage regulation

[17] and is not outlined in detail here. The resulting averaged model is given by

$$
G(s)=I_{s c}(s)=\frac{V_{c s c}(s)-V_{s c}(s)}{s l_{s c}+r_{s c}} .
$$

A variable change is proposed in the above model given by

$$
V_{m s c}(s)=V_{c s c}(s)-V_{s c}(s) \text {. }
$$

The resulting converter model with the variable change is given by

$$
G(s)=\frac{I_{s c}(s)}{V_{m s c}(s)}=\frac{1}{s l_{s c}+r_{s c}} .
$$

\section{THE ADRC SCHEME FOR VOLTAGE REGULATION}

The ADRC based voltage regulation is schematically represented as shown in Fig. 2. The voltage regulation part employs the PI+ESO observer for estimating the disturbance, which causes the deviation of voltage from its nominal value. The disturbance in the case of system having high renewable penetration ( as is the case considered here) will be difference of load current demanded from grid and the source current constituted by the renewable generation. Therefore this disturbance current signifies the deficit or surplus power in the grid which should be catered by the storage system. The current control block in Fig. 2 forms the inner loop that acts on the disturbance reference generated by the observer and ensures that the required current is delivered to the grid by the storage system to compensate the load mismatch.

\section{A. $P I+E S O$}

The modelling of the ESO requires the system model to be defined first. The grid voltage equation for the system shown in Fig. 2 is written as

$$
C \frac{d v_{b u s}}{d t}=\bar{\xi}+i_{s c}
$$


where $C$ represents the equivalent capacitance of the DC bus, and $\bar{\xi}$ represents the disturbance in the DC grid. In the system considered here $\bar{\xi}$ is defined as

$$
\bar{\xi}=i_{r s}-i_{\text {load }}
$$

where $i_{r s}$ represents the current supplied to the grid by renewable sources and $i_{\text {load }}$ represents the current drawn by loads from the grid. Since both the generation and load demands are non deterministic $\bar{\xi}$ is also non deterministic. by

Equation (4) can be rewritten as a perturbed system given

$$
\frac{d v_{b u s}}{d t}=\xi+k i_{s c}
$$

where $k=\frac{1}{C}$ and $\xi$ is $\frac{\bar{\xi}}{C}$. Since the objective is to incorporate the disturbance as an extended state of the system a model for the same is proposed to define an augmented system. The disturbance can be modelled as

$$
\frac{d^{m} \xi}{d t} \approx 0
$$

where $m$ is large enough such that it an accommodate the disturbance model. Additionally, $\xi$ is also assumed to be class $C_{m-1}$ i.e. the first m-1 derivative of $\xi$ exist and are continuous. Therefore the state space representation of the augmented system using the disturbance model is given by

$$
\begin{aligned}
\dot{\mathbf{x}} & =\mathbf{A} \mathbf{x}+\mathbf{b} i_{s c} \\
v_{\text {bus }}=y & =\mathbf{c} \mathbf{x}
\end{aligned}
$$

with

$$
\begin{aligned}
& \mathbf{x}=\left[\begin{array}{c}
x_{1} \\
x_{2} \\
\cdot \\
\cdot \\
\cdot \\
x_{n-1} \\
x_{n}
\end{array}\right] \\
& \mathbf{A}=\left[\begin{array}{ccccccc}
0 & 1 & 0 & . & . & . & 0 \\
0 & 0 & 1 & . & . & . & 0 \\
\cdot & . & . & . & & & . \\
. & . & . & & . & & . \\
. & . & . & & & . & . \\
0 & 0 & 0 & . & . & . & 1 \\
0 & 0 & 0 & . & . & . & 0
\end{array}\right], \quad \mathbf{b}=\left[\begin{array}{c}
k \\
0 \\
\cdot \\
. \\
. \\
0 \\
0
\end{array}\right]
\end{aligned}
$$

where $\mathbf{x} \in \mathbb{R}^{m+1}$ is the state of system such that $x_{1}=v_{\text {bus }}$, $x_{2}=\xi$ and $x_{n}=\frac{d^{m-1} \xi}{d t^{m-1}}$ with $m$ as in (7). $\mathbb{R}$ is the set of real numbers.

The system model defined in (8) is then used for the observer. The ESO structure is the same as that of the Luenberger observer with the additonal states in the system contributed by the disturbance. The ESO observer is then given by

$$
\dot{\hat{\mathbf{x}}}=\mathbf{A} \hat{\mathbf{x}}+\mathbf{B} i_{s c}+\mathbf{L}(y-\hat{y})
$$

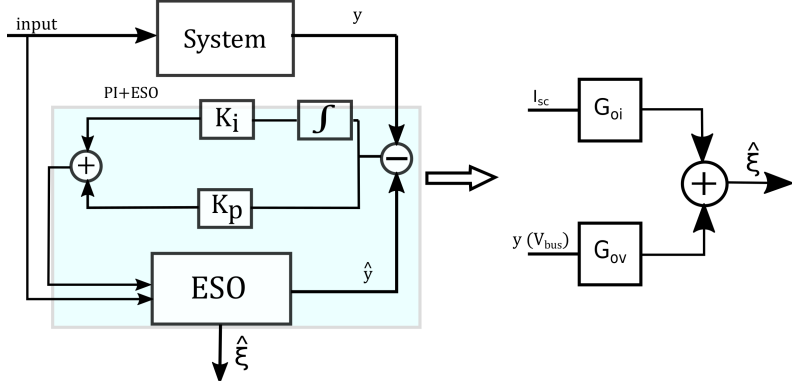

Fig. 3. PI+ESO schematic

where $\hat{\mathbf{x}}$ represents the estimation of states shown in (9), $\hat{y}$ is the estimated value of $v_{b u s}$ by ESO, $y$ is measured value of $v_{\text {bus }}$ and $\mathbf{L}$ is the gain of observer.

The PI+ESO is realised by augmenting an additional state to the system in (11). This additional state is the integral of ESO tracking error, which can arise due to modelling uncertainties, as shown schematically in Fig.3. This ensures robustness to parameter uncertainties. The integral action is added as an input to all the states. The proposed PI+ESO can therefore, be represented in the state space form as

$$
\left[\begin{array}{c}
\dot{\hat{\mathbf{x}}} \\
\hat{\hat{d}}
\end{array}\right]=\left[\begin{array}{cc}
\mathbf{A} & 1 \\
0 & 0
\end{array}\right]\left[\begin{array}{c}
\hat{\mathbf{x}} \\
\hat{d}
\end{array}\right]+\left[\begin{array}{c}
\mathbf{B} \\
0
\end{array}\right] i_{s c}+\left[\begin{array}{c}
\mathbf{L} \\
I
\end{array}\right](y-\hat{y})
$$

where $\hat{d}$ represent the additional state which is integral of the observer estimation error $(\dot{\hat{d}}=y-\hat{y}), \mathbf{A}$ and $\mathbf{B}$ are same as that in (10), and $I$ is the gain of integral part. Again the system represented in (12) is the same structure as that of the Luenberger observer and as such the gain scheduling for the observer can be done using the standard pole placement method. It should be noted that the PI+ESO forms a system with two inputs, $i_{s c}$ and $\mathrm{y}\left(v_{\text {bus }}\right)$, resulting in the equivalent block diagram representation as in Fig.3 with the transfer function $G_{o i}$ and $G_{o v}$ respectively for the inputs. The output from proposed observer will be the disturbance estimation $(\hat{\xi})$.

\section{B. Control law}

Once the estimate of the disturbance is obtained a control law has to be defined to ensure the rejection of this disturbance. As discussed before the current control loop in Fig.2 when provided with the suitable reference in the form of $i_{\text {scref }}$ will ensure the disturbance rejection. The control action is therefore defined as

$$
i_{\text {scref }}=\frac{1}{k}\left[\dot{v}_{b u s r}+k_{p v} e_{b}+k_{i v} \int e_{b}-\hat{\xi}\right] .
$$

where, $v_{\text {busr }}$ is the reference value of the grid voltage, $e_{b}$ is the tracking error of the grid voltage given by ( $v_{b u s r}-$ $\left.v_{b u s}\right), \hat{\xi}$ is the estimated disturbance and $k_{p v}, k_{i v}$ represents gains. It should be noted that $e_{b}$ is not to be confused with the estimation error of the observer $(y-\hat{y})$. Substituting (13) in (6) results in

$$
\dot{e}_{b}+k_{p v} e_{b}+k_{i v} \int e_{b}=\hat{\xi}-\xi .
$$




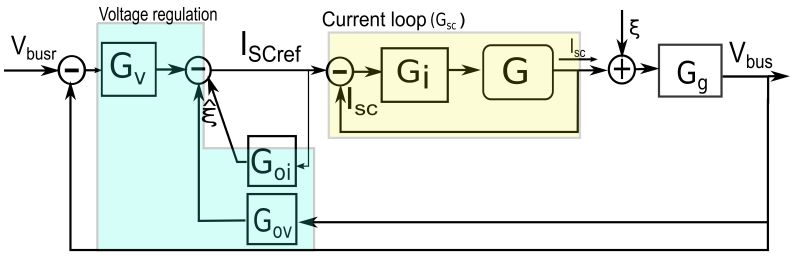

Fig. 4. An equivalent gain block based representation of the ADRC acheme

Employing a disturbance observer discussed above with negligible estimation error, the right hand side of (14) tends to zero resulting in (14) being dominated by the dynamics of the following characteristic polynomial

$$
p_{e b}=s^{2}+k_{p v} s+k_{i v} .
$$

A suitable selection of $k_{p v}$ and $k_{i v}$ will ensure that the voltage regulation loop be Hurwitz with required dynamical characteristics ensuring that the tracking error $e_{b}$ will lie in the vicinity of zero, disregarding the disturbance function $\xi$. It should be noted that in the control law defined by (13) there is a feedback back part formed by $k_{p v}$ and $k_{i v}$ which is incorporated in block $c_{f b}$ in Fig.2 and a feed forward path formed by $\dot{v}_{\text {busr }}$. In the case of the voltage regulation of grid connected system presented in this work the reference grid voltage is constant resulting in the feed forward term being zero and hence not shown in Fig.2.

\section{IV. $H_{\infty}$ AND $H_{2}$ NORM ANALYSIS FOR ADRC SCHEME}

The effectiveness and improvement in performance brought about by the ADRC scheme based voltage regulation is quantified using the $\mathrm{H}_{\infty}$ and $\mathrm{H}_{2}$ norms of the system. In order that such an analysis be carried out, the transfer function of the system from disturbance input $(\xi)$ to the output $\left(v_{b u s}\right)$ needs to be evaluated. The Fig.4 represents the equivalent block diagram representation of the control scheme presented in Fig.2. The PI+ESO in Fig.2 has been replaced with its equivalent block diagram of a two input system as shown in Fig.3. Similarly $G_{i}$ represent the transfer function of the controller, $P I_{I}$, in the current control loop, $G$ is the transfer function of the DC-DC converter given in (3), $G_{g}$ is the transfer function of the grid and $G_{v}$ is the transfer function of block $c_{f b}$ which are all shown in Fig.2. The transfer function from the $\xi$ to output $V_{b u s}$ is then given by block diagram reduction to be

$$
G_{s y s d}=\frac{G_{i} G G_{g}\left(G_{v}+G_{o v}\right)}{1+G_{o i}+G_{i} G\left(G_{g}\left(G_{v}+G_{o v}\right)+1+G_{o i}\right)} .
$$

The performance of the ADRC based voltage regulation will be compared with the conventional dual loop scheme presented in Fig.5. The block gains $\left(G_{v}, G_{i}, G, G_{g}\right)$ represented in Fig.5 are same as that in Fig.4. The disturbance to output transfer function of the dual loop structure is then given by

$$
G_{\text {sysnd }}=\frac{G_{g}\left(G_{1} G+1\right)}{1+G_{i} G\left(G_{g} G_{v}+1\right)} .
$$

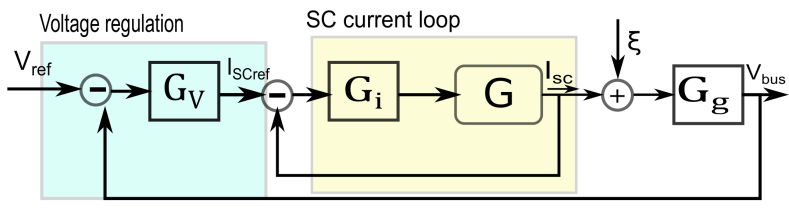

Fig. 5. Dual loop control architecture.

TABLE I

SYSTEM NORMS FOR THE ADRC AND DUAL LOOP CONTROL SCHEMES

\begin{tabular}{|l||l||l|}
\hline System & $\mathrm{H}-\infty$ & $\mathrm{H} 2$ \\
\hline$G_{\text {Sysd }}$ & 0.6359 & 12.5535 \\
$G_{\text {Sysnd }}$ & 1.1161 & 14.9959 \\
\hline
\end{tabular}

First the norms are defined. The $H_{\infty}$ norm of any system with transfer function $T(s)$ is given by [18]

$$
\|T(s)\|_{\infty}=\sup _{\omega}|T(j \omega)|
$$

while the $H_{2}$ norm is given by [18]

$$
\|T(s)\|_{2}=\left(\frac{1}{2 \pi} \int_{-\infty}^{\infty}|T(j \omega)|^{2} d \omega\right)^{\frac{1}{2}}
$$

The calculated values of these norms for the system $G_{\text {sysd }}$ and $G_{\text {sysnd }}$ are shown in Table.I. The values of $H_{\infty}$ and $H_{2}$ norms show a reduction in the ADRC scheme in comparison to the dual loop architecture. The $\mathrm{H}-\infty$ infinity norm provides an upper bound on the $\|y\|_{2}$ norm of the output for a square integrable input signal [18]. This $\|y\|_{2}$ norm implies energy associated with the output signal. Therefore the reduction in the $H_{\infty}$ norm with the ADRC scheme translates to a reduction in the energy level of the disturbance rejection response in comparison to the conventional voltage regulation architecture in Fig.5 resulting in an improved stability of the system. Similarly the $H_{2}$ norm provides an upper bound on the $\|y\|_{\infty}$ norm of the output for a square integrable input signal [18]. The $\|y\|_{\infty}$ norm represents the least upper bound of the absolute value of the signal. This ensures a reduction in the peak value of the disturbance response. This comparison of the performance with $H_{\infty}$ and $H_{2}$ norm is valid for the class of signals which are square integrable. In the case of microgrid this is more relevant as the variation in renewable generation is non deterministic and not known a priori. As a result such a norm based analysis provides more general understanding on the system behaviour.

It should be noted that the ADRC scheme will not provide an improved performance over a classical feed forward scheme shown in Fig.6 where the disturbance can be measured and feed forwarded [19]. Nevertheless in a system like microgrid with distributed generation this can lead to large resource allocation in terms of high bandwidth communication and sensor allocation to capture the entire disturbance profile. The ADRC scheme can therefore provide an alternative which is a definite improvement over the conventional dual loop architecture as evidenced from above but with less resource 


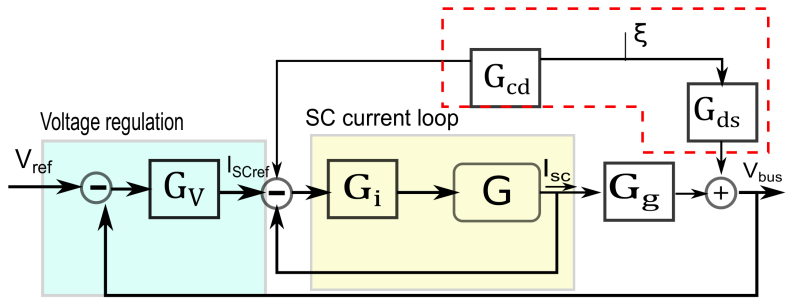

Fig. 6. Feed forward scheme for disturbance rejection

requirements. The proposed scheme can easily be implemented in any digital controller to generate a disturbance estimation.

\section{Simulation Results}

In this section the implementation of the ADRC scheme for voltage regulation applied to the converter system shown in Fig. 1 and the results obtained will be discussed. For the converter system the ADRC scheme is developed considering a second order disturbance model. Therefore, the value of $m$ in (6) will be 2 . The second order model is considered as most of the disturbance occurring in the grids can be sudden variation (step) or gradual variation (ramp). The first step in the design process is to fix the gains of the PI controller in the current control loop. This is the fastest loop and the gains are designed such that the loop has a settling time of $2 \mathrm{~ms}$. The next step is defining the gains of observer $\mathbf{L}$ and $I$ which will be determined using pole placement technique. They are designed such that observer settling time is ten times that of the inner current loop thereby ensuring that the current loop output will be seen as a steady state signal for the observer. Finally the $k_{p v}$ and $k_{i v}$ values of $C_{f b}$ are decided such that the voltage regulation loop error dynamics settles within 40 ms. This ensures that the observer appears in steady state for the voltage regulation loop.

The simulation models were developed in Matlab Simulink. A complete model of the system including passive components, power electronics devices like IGBT, data acquisition filters were considered in the model using components from the powerlib library of Simulink.

The Fig.7 shows the PI+ESO observer disturbance estimation. The disturbance reference (red) shows the deficit current in the grid to be supplied by the ESS. The current estimated by the observer is also shown in Fig.7. It can be seen that the observer estimation of the disturbance is greater than the reference. This is due to the non-ideal nature of the devices and components considered in the simulation models. The inductors, capacitors and IGBTs are modelled as elements with losses in the simulation model and hence the current supplied by the ESS has to cater these losses resulting in a higher disturbance estimate by PI+ESO. Though not modelled, the $\mathrm{PI}+\mathrm{ESO}$ is capable of estimating these losses.

The Fig. 8 shows grid voltage profiles under the disturbances when using the ADRC scheme. In Fig.8 the performance of the ADRC scheme (blue) is compared with a conventional dual loop voltage regulation (orange) scheme for power converters

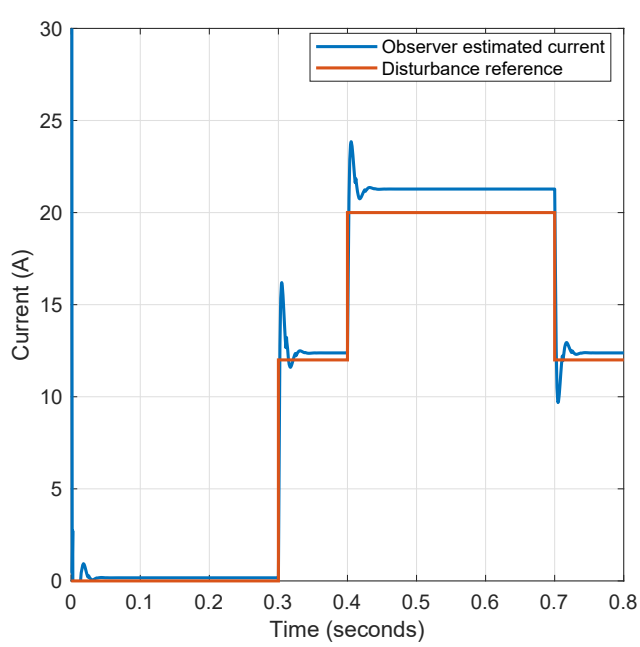

Fig. 7. Disturbance estimation of the PI+ESO

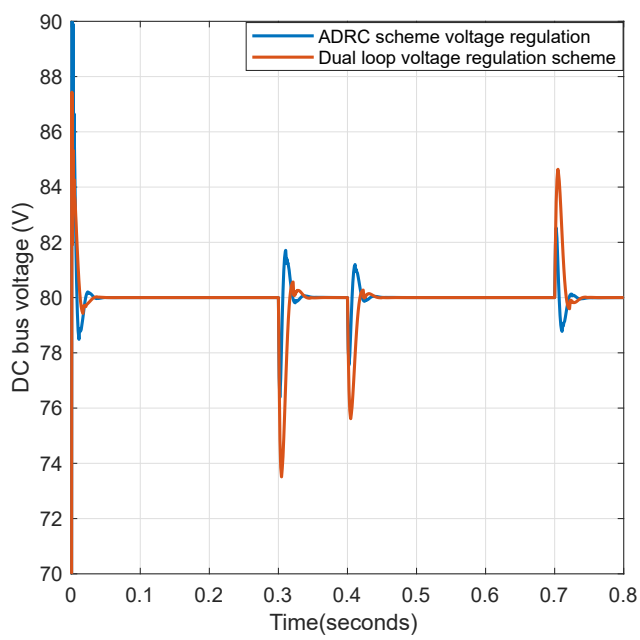

Fig. 8. Voltage regulation performance of the ADRC and the standard dual loop control architecture

shown in Fig.5. The improvement in the voltage regulation functionality can be observed here with the deviation in grid voltage from the nominal value lesser in the case of ADRC scheme compared to conventional dual loop architecture. Since the controller gains considered in both architecture are same, the improvement in performance can be attributed to the disturbance estimation by the observer. The simulation results obtained is coherent with norm based analysis made in the previous section with reduced overshoot of response and improved dynamics as evidenced by the reduced values of the norms.

The Fig.9 shows observer estimation under parameter variation. The grid in this work has been modelled as a capacitor. The design of the observer was done considering a constant value for the capacitor. In the real scenario this may not be case. The capacitor value can change depending on the operation condition in the grid with number of loads and sources connected at any particular instant. The robustness of 


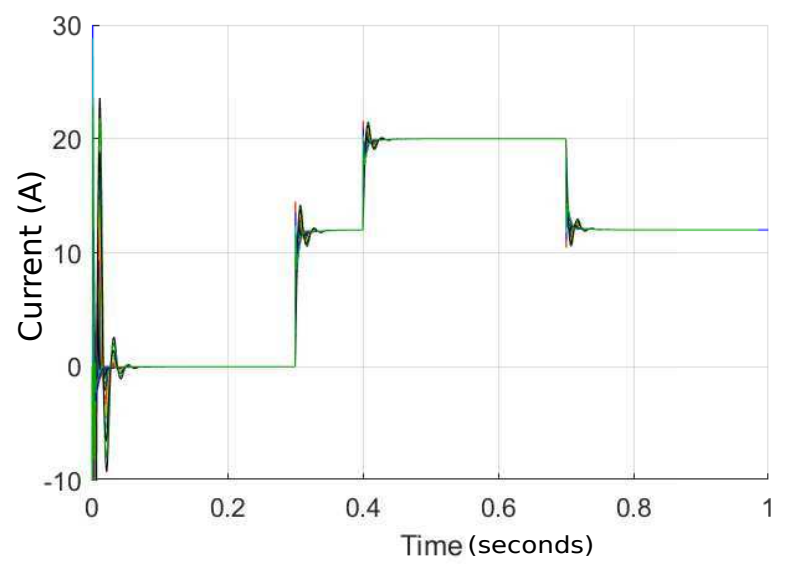

Fig. 9. Load disturbance estimation of the observer under varying DC-link capacitor values.

observer estimation is highlighted through the plot in Fig.9. It shows the observer estimation of the disturbance under varying values of the grid capacitance. The plots are generated keeping the disturbance constant and varying the equivalent grid capacitance value within a range of $\pm 90 \%$ of the nominal value (50 samples are considered). It can be observed that under the variation of grid capacitance value, the estimation of the observer remains constant showing the effectiveness of the PI+ESO under parameter uncertainty. It can be noted that in Fig.9 there are some large oscillations in the estimation of the disturbance. This arises because the capacitance variation in the grid is set at $\pm 90 \%$ and as a result with very low grid capacitance case (weak grid), which occurs near $-90 \%$, the variation in grid voltage is high for disturbances resulting in large oscillations in the estimation. This may not be a realistic case but such a variation was used for simulations to highlight the robustness of observer.

\section{CONCLUSION}

The ADRC scheme based voltage regulation has exhibited an improved performance over the standard dual loop control architecture for voltage regulation in power converters. In systems with disturbances that are non-deterministic in nature like grid connected systems this scheme can find increased application. This work has only aimed at addressing some initial aspects of the implementation of such observers in grid connected systems mainly in terms of investigating suitability of such observers in grid connected systems and their improvement in performance. There is scope for more work in this area especially a formal analysis on system stability. Also more work can be done in the physical implementation of such systems in a real set-up to identify practical implementation issues.

\section{ACKNOWLEDGMENTS}

This work is done as part of project which has received funding from the European Union's Horizon 2020 research and innovation programme under the Marie Skłodowska Curie grant agreement No 675318 (INCITE).

This work has been partially funded by the MINECO/FEDER project DPI2015-69286-C3-2-R. This work is supported by the Spanish State Research Agency through the Mara de Maeztu Seal of Excellence to IRI (MDM-2016-0656).

This work is partially funded by AGAUR of Generalitat de Catalunya through the Advanced Control Systems (SAC) group grant (2017 SGR 482).

\section{REFERENCES}

[1] Paul Denholm, Erik Ela, Brendan Kirby, and Michael Milligan. The Role of Energy Storage with Renewable Electricity Generation The Role of Energy Storage with Renewable Electricity Generation. Contract, NREL/(January):1-53, 2010.

[2] Haisheng Chen, Thang Ngoc Cong, Wei Yang, Chunqing Tan, Yongliang $\mathrm{Li}$, and Yulong Ding. Progress in electrical energy storage system: A critical review. Progress in Natural Science, 19(3):291-312, 2009.

[3] Laureano Piris-Botalla, Germn G. Oggier, Andrs M. Airabella, and Guillermo O. Garca. Extending the soft-switching operating range of a bi- directional three-port dc-dc converter. Revista Iberoamericana de Automtica e Informtica Industrial RIAI, 13(1):127 - 134, 2016.

[4] JA Peças Lopes, CL Moreira, and AG Madureira. Defining control strategies for microgrids islanded operation. IEEE Transactions on power systems, 21(2):916-924, 2006.

[5] Chengshan Wang, Xialin Li, Li Guo, and Yun Wei Li. A nonlineardisturbance-observer-based dc-bus voltage control for a hybrid ac/dc microgrid. IEEE Transactions on Power Electronics, 29(11):6162-6177, 2014.

[6] Frede Blaabjerg, Remus Teodorescu, Marco Liserre, and Adrian V Timbus. Overview of control and grid synchronization for distributed power generation systems. IEEE Transactions on industrial electronics, 53(5):1398-1409, 2006.

[7] Adrian Timbus, Marco Liserre, Remus Teodorescu, Pedro Rodriguez, and Frede Blaabjerg. Evaluation of current controllers for distributed power generation systems. IEEE Transactions on power electronics, 24(3):654-664, 2009.

[8] Sayed Ali Khajehoddin, Masoud Karimi-Ghartemani, Praveen K Jain, and Alireza Bakhshai. Dc-bus design and control for a single-phase gridconnected renewable converter with a small energy storage component. IEEE Transactions on Power Electronics, 28(7):3245-3254, 2013.

[9] Josep M Guerrero, Juan C Vasquez, José Matas, Luis García De Vicuña, and Miguel Castilla. Hierarchical control of droop-controlled ac and dc microgridsa general approach toward standardization. IEEE Transactions on industrial electronics, 58(1):158-172, 2011.

[10] Makoto Hagiwara and Hirofumi Akagi. An approach to regulating the dc-link voltage of a voltage-source btb system during power line faults. IEEE transactions on industry applications, 41(5):1263-1271, 2005.

[11] B. Parkhideh and S. Bhattacharya. Vector-controlled voltage-sourceconverter-based transmission under grid disturbances. IEEE Transactions on Power Electronics, 28(2):661-672, Feb 2013.

[12] F Bakhshande and D Söffker. Proportional-integral-observer: A brief survey with special attention to the actual methods using acc benchmark. IFAC-PapersOnLine, 48(1):532-537, 2015.

[13] E Davison. The output control of linear time-invariant multivariable systems with unmeasurable arbitrary disturbances. IEEE Transactions on Automatic Control, 17(5):621-630, 1972.

[14] CD Johnson. Theory of distrubance-accommodating controllers. In Control and Dynamic Systems, volume 12, pages 387-489. Elsevier, 1976.

[15] B Wojciechowski. Analysis and synthesis of proportional-integral observers for single-input-single-output time-invariant continuous systems. Gliwice, Poland, 1978.

[16] Jianxing Liu, Sergio Vazquez, Ligang Wu, Abraham Marquez, Huijun Gao, and Leopoldo G Franquelo. Extended state observer-based slidingmode control for three-phase power converters. IEEE Transactions on Industrial Electronics, 64(1):22-31, 2017.

[17] Robert W Erickson and Dragan Maksimovic. Fundamentals of power electronics. Springer Science \& Business Media, 2007.

[18] J Doyle, B Frances, A Tannenbaum, and KL Moore. Feedback control theory. IEEE Transactions on Automatic Control, 39(7):1532-1533, 1994.

[19] Charles L Phillips and Royce D Habor. Feedback control systems. Simon \& Schuster, 1995. 